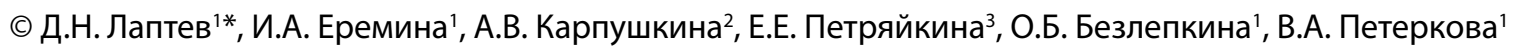

1Национальный медицинский исследовательский центр эндокринологии, Москва

${ }^{2}$ Благотворительный фонд развития филантропии «КАФ», Москва

зРоссийская детская клиническая больница, Москва

АКТУАЛЬНОСТЬ. Распространенность сахарного диабета 1 типа (СД1) в детском возрасте увеличивается с каждым годом. Подростковый возраст является наиболее трудным в отношении достижения оптимального метаболического контроля СД1. Телемедицина уже продемонстрировала эффективность у детей с этим заболеванием, однако исследований среди подростков проведено недостаточно. Использование мобильных приложений для диабета может быть связано с лучшим гликемическим контролем у пациентов с СД1.

ЦЕЛЬ. Оценить эффективность и безопасность модели медицинской помощи подросткам с СД1 с применением дистанционного консультирования и мобильного приложения.

МАТЕРИАЛЫ И МЕТОДЫ. В Исследование включались подростки в возрасте $\geq 14$ и <18 лет, с длительностью СД1 $>3$ мес. Длительность исследования составляла 26 нед. Проведено 3 очных и не менее 4 дистанционных визитов с использованием мобильного приложения. Всем пациентам проводились стандартный осмотр и антропометрия, исследование $\mathrm{HbA}_{1 c^{\prime}}$ регистрация и анализ показателей, оценка и коррекция проводимого лечения. Исходно и в конце исследования оценивалось качество жизни подростков. В конце исследования проводился опрос подростков и лечащих врачей в отношении оценки программы.

РЕЗУЛЬТАТЫ. Были включены 56 пациентов, 7 подростков прервали участие. Уровень НbА

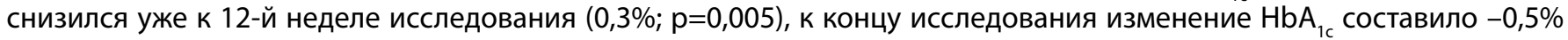
( $<<0,001)$. Отмечалось статистически значимое увеличение процентов измерений глюкозы в целевом диапазоне (+5,3 п.п.; $p=0,016)$ и снижение вариабельности глюкозы крови $(-3,1$ п.п.; $p=0,015)$. Отмечено статистически значимое улучшение как совокупной оценки качества жизни пациентами (+2,9 балла; $p=0,008)$, так и отдельных составляющих ее показателей: отношение к диабету $(+3,0$ балла; $p=0,049)$, отношение к лечению (+4,6 балла; $p=0,010)$ и общение с окружающими (+4,5 балла; $\mathrm{p}=0,015)$. Большинство врачей и пациентов положительно оценили участие в исследовании. Частота нежелательных явлений значимо не изменилась в процессе исследования по сравнению с исходным уровнем.

ЗАКЛЮЧЕНИЕ. Дистанционное консультирование с использованием мобильного приложения для управления диабетом является безопасным и эффективным в отношении гликемического контроля и качества жизни подходом у подростков с СД1, обеспечивающим удобство и быстроту взаимодействия.

КЛЮЧЕВЫЕ СЛОВА: сахарный диабет 1 типа; подростки; дистанционное наблюдение; мобильное приложение; телемедицина

\title{
REMOTE MONITORING OF ADOLESCENTS WITH TYPE 1 DIABETES MELLITUS USING A MOBILE APPLICATION
}

(c) Dmitry N. Laptev1*, Irina A. Eremina', Anna V. Karpushkina², Elena E. Petryaykina³, Olga B. Bezlepkina', Valentina A. Peterkova

'Endocrinology Research Centre, Moscow

${ }^{2}$ The "CAF" Charitable Foundation for Philanthropy, Moscow

${ }^{3}$ Pirogov Russian National Research Medical University, Moscow

BACKGROUND: The prevalence of type 1 diabetes mellitus (T1DM) in childhood is increasing every year. Adolescence is the most challenging age for achieving optimal metabolic control of T1DM. Telemedicine has already been shown to be effective in children with the condition, but there are not enough studies in adolescents. The use of mobile apps may be associated with better glycemic control in patients with type 1 diabetes.

AIMS: To assess the effectiveness and safety of a model of medical care for adolescents with type 1 diabetes using remote counseling and a mobile application. 
MATERIALS AND METHODS: Were included adolescents aged $\geq 14$ and 18 years with a T1DM duration $>3$ months, a glycated hemoglobin level $\left(\mathrm{HbA}_{1 c}\right)>7 \%$. The duration of the study was 26 weeks. There were 3 face-to-face and at least 4 remote visits using a mobile application. All patients underwent standard examination and anthropometry, study of $\mathrm{HbA}_{1 c^{\prime}}$ registration and analysis of indicators, assessment and correction of the treatment. The quality of life of adolescents was assessed at baseline and at the end of the study. Adolescents and physicians were interviewed about program evaluation.

RESULTS: 56 patients were included, 7 adolescents withdrew. $\mathrm{HbA}_{1 \mathrm{c}}$ significantly decreased by the 12th week of the study $(-0.3 \% ; p=0.005)$, by the end of the study the change in $\mathrm{HbA}_{1 c}$ was $-0.5 \%(p<0.001)$. There was an increase in the percentage of glucose measurements in the target range $(+5.3 \mathrm{pp} ; \mathrm{p}=0.016)$ and a decrease in blood glucose variability $(-3.1 \mathrm{pp}$; $p=0.015)$. There was a significant improvement in both the total assessment of the quality of life by patients ( 2.9 points; $p=0.008)$ and individual components of its indicators: attitude to diabetes $(+3.0$ points; $p=0.049)$, attitude to treatment $(+4.6$ points; $p=0.010)$ and communication with others ( +4.5 points; $p=0.015)$. The majority of doctors and patients assessed their participation in the study positively. The incidence of adverse events did not change significantly during the study from baseline.

CONCLUSION: Remote counseling using a mobile app is a safe and effective approach for adolescents with T1DM in terms of glycemic control and quality of life, and provides convenience and speed of interaction.

KEYWORDS: type 1 diabetes mellitus; adolescents; remote monitoring; mobile application; telemedicine

\section{АКТУАЛЬНОСТЬ}

Сахарный диабет 1 типа (СД1) - это хроническое социально значимое заболевание, распространенность которого в детском возрастеувеличивается с каждым годом [1]. Так, в Российской Федерации, по данным Федерального регистра пациентов с сахарным диабетом, распространенность СД1 среди детей в возрасте до 14 лет с 2013 по 2016 г. увеличилась с 81 до 93,5 случая на 100000 детского населения, а подростков в возрасте 15-17 лет - с 212,8 до 221,7 случая на 100000 подросткового населения [2].

Наиболее трудным с точки зрения достижения оптимального метаболического контроля СД1 является подростковый возраст, что определяется биологическими и психосоциальными изменениями, связанными с половым созреванием, создающими нехарактерные для другого возраста потребности и проблемы для самих подростков с диабетом и их семей [3]. В результате в подростковом возрасте наблюдается наиболее высокий уровень гликированного гемоглобина $\left(\mathrm{HbA}_{1 c}\right)$ [2]. При этом по сравнению со взрослыми метаболический контроль в подростковом возрасте, по-видимому, имеет большее влияние на риск микрососудистых осложнений в более позднем возрасте [4], что указывает на необходимость более тщательного контроля диабета.

В последнее время на фоне происходящей информатизации общества в целом и благодаря своей доступности и удобству широкое распространение получают различные мобильные приложения для контроля диабета. С помощью таких приложений пациенты с СД1 и их родители могут собрать все данные по самоконтролю в одном месте и получить совет своего лечащего врача по инсулинотерапии, подсчету углеводов или настройке устройств. Использование мобильных приложений может быть связано с лучшим гликемическим контролем у пациентов с СД1 [5].

Препятствиями для использования мобильных приложений для управления диабетом являются недостаточная осведомленность о подходящих продуктах и уверенность в том, что приложение не принесет пользы [5]. Кроме того, определять эффективность могут функциональные возможности, так, среди характеристик мобиль- ных приложений, которые связаны с лучшим уровнем $\mathrm{HbA}_{1 c^{\prime}}$ - наличие структурированного представления данных самоконтроля [6].

Телемедицина уже продемонстрировала свою эффективность у детей с СД1 [7], однако исследований у подростков проведено недостаточно. Поскольку подавляющее большинство подростков не достигают целевого гликемического контроля, принимая во внимание особые потребности в этом возрасте, целесообразно оценить эффективность дистанционной модели наблюдения с применением мобильного приложения, чтобы повысить эффективность медицинской помощи.

\section{ЦЕЛЬ}

Оценить эффективность и безопасность модели медицинской помощи подросткам с СД1 с применением дистанционного консультирования и мобильного приложения.

\section{МАТЕРИАЛЫ И МЕТОДЫ}

\section{Дизайн исследования}

Многоцентровое проспективное открытое неконтролируемое клиническое исследование было проведено в 10 медицинских организациях, находящихся на территории г. Москвы и оказывающих медицинскую помощь в амбулаторных условиях. В исследование были включены подростки в возрасте $\geq 14$ и <18 лет, с длительностью СД1 $>3$ мес, получающие интенсифицированную инсулинотерапию (путем множественных инъекций инсулина - МИИ или непрерывной подкожной инфузии инсулина - НПИИ).

Процедуры исследования и регистрация показателей Длительность исследования составляла 26 нед, исследование включало два периода: период введения и основной период. Длительность периода введения составляла 2 нед, в течение которых проводилась только регистрация показателей гликемии по данным исследовательского глюкометра без мобильного приложения и дистанционного наблюдения. Длительность основного периода составила 24 нед, в течение которых 
было проведено три очных (исходно, через 12 и 24 нед) и не менее 4 дистанционных визитов (через 4, 8, 16, 20 нед) с использованием мобильного приложения.

Всем пациентам на очных визитах проводились стандартный осмотр и антропометрия, осуществлялись исследование уровня $\mathrm{HbA}_{1 с}$ методом реакции агглютинации моноклональных антител на анализаторе DCA Vantage Analyzer (Siemens, Германия), регистрация и анализ показателей гликемии и суточных доз инсулина, оценка и коррекция проводимого лечения, давались рекомендации по самоконтролю и наблюдению. Исходно и в конце исследования проводилась оценка качества жизни подростков. Помимо этого, в конце исследования был проведен опрос подростков и лечащих врачей в отношении оценки программы дистанционного наблюдения с применением мобильного приложения.

\section{Мобильное приложение OneTouch Reveal (OT Reveal)}

Bce пациенты были обеспечены глюкометром OneTouch Select Plus Flex с возможностью автоматической передачи данных на смартфон в специализированное мобильное приложение ОT Reveal для управления СД и обучены его использованию. Мобильное приложение OT Reveal представляет собой дневник самоконтроля в электронном формате с автоматическим построением структурированных отчетов и возможностью направлять их в электронном виде медицинскому работнику. Приложение предназначено для граждан Российской Федерации и доступно на русском языке.

Пациенту или его законному представителю было рекомендовано выполнять измерения глюкозы крови с частотой не менее 4 раз в сутки (перед едой, через 2 ч после еды, перед сном, периодически ночью) с помощью совместимого глюкометра. Помимо этого, пациентам было рекомендовано вносить в мобильное приложение данные о времени и количестве принятых углеводов (в хлебных единицах и граммах), времени и дозе введенного инсулина, времени и характере других событий (физические нагрузки, гипогликемия и др.). При использовании непрерывного мониторинга глюкозы (НМГ) пациент также мог самостоятельно вносить данные по уровню гликемии в мобильное приложение.

\section{Дистанционное консультирование}

Дистанционное консультирование проводилось лечащими врачами, наблюдавшими пациента в амбулаторных условиях, на основании данных электронных структурированных отчетов, формируемых в приложении OneTouch Reveal. Принимая во внимание полученные данные, лечащий врач дистанционно консультировал пациента и при необходимости корректировал проводимую терапию/давал рекомендации по самоконтролю.

Оценка качества жизни и дистанционного

консультирования с применением мобильного приложения ОT Reveal

Оценка качества жизни детей и родителей проводилась с использованием специализированных опросников. Опросник состоит из 28 элементов (ситуаций), составляющих 5 модулей: Диабет, Лечение-І, Лечение-II, Беспокойство, Общение. В опроснике пациентам предлагается оценить, насколько затруднительными для них были различные ситуации в течение последнего месяца. Каждый элемент может быть оценен по 5-балльной шкале от 0 (никогда) до 4 (почти всегда). Для подсчета полученных результатов баллы по каждому элементу трансформируются в обратном порядке по шкале от 0 до 100 следующим образом: $0=100,1=75,2=50,3=25,4=0$. Затем рассчитывается средний балл по всем элементам в целом и по каждому разделу отдельно. Чем выше балл, тем лучше качество жизни пациента.

Оценка программы дистанционного консультирования с мобильным приложением проводилась с использованием специально разработанных опросников, в которых пациентам и врачам было предложено охарактеризовать удобство, сложности и преимущества использованного в исследовании информационного подхода. Опросник для врачей включал в себя 8 вопросов, 5 из которых необходимо было оценить по 5-балльной шкале от 1 (совсем не согласен) до 5 (полностью согласен) и на 3 ответить «да» или «нет». Опросник для подростков включал в себя 10 вопросов, 7 из которых необходимо было оценить по 5-балльной шкале от 1 (совсем не согласен) до 5 (полностью согласен) и на 3 ответить «да» или «нет».

\section{Основной исход исследования}

Изменение $\mathrm{HbA}_{1 с}$ к концу исследования по сравнению с исходным уровнем и доля пациентов, достигших $\mathrm{HbA}_{1 с}$ менее 7,0 и 7,5\% к концу исследования.

\section{Дополнительные исходы исследования}

Изменение к концу исследования по сравнению с исходным уровнем: 1) показателей гликемического контроля: средний уровень гликемии, вариабельность гликемии (SD и CV), процент измерений в диапазоне от 3,9 до 10,0 , менее 3,9 и более 10,0 ммоль/л; 2) показателей качества жизни; 3) острых осложнений СД1: частота эпизодов диабетического кетоацидоза (ДКА), тяжелой гипогликемии и гипергликемии с кетозом.

\section{Этическая экспертиза}

Исследование одобрено локальным комитетом по этике ФГБУ «НМИЦ эндокринологии» Минздрава России (протокол номер №11 от 14.10.2015). До включения в исследование законные представители пациентов подписали информированное согласие на участие в нем.

\section{Статистический анализ}

Размер выборки предварительно не рассчитывался. Обработка и анализ статистических данных проводились в программах Statistica 8.0 (StatSoft, США), MS Excel 2010 (Microsoft, США). Количественные данные представлены в виде среднего значения и его стандартного отклонения M (SD) в случае нормального распределения признака или в виде медианы и интерквартильного размаха Ме (25-75 перцентиль) - в случае распределения признака, отличного от нормального; качественные данные представлены в виде абсолютных значений (n) и/или частот (\%), данные о ДКА, кетозах и тяжелой гипогликемии представлены также в виде частоты эпизодов в пересчете на 1 пациента в год. Различие между количественными признаками в зависимых выборках оценивалось с помощью Т-критерия Вилкоксона, в независимых выборках - 
Таблица 1. Клиническая характеристика участников исследования

\begin{tabular}{lc}
\hline \multicolumn{1}{c}{ Показатель $(\mathbf{n = 4 9 )}$} & Значение \\
\hline Пол, м/ж & $23 / 26$ \\
Возраст, лет & $15,6(14,7-16,8)$ \\
Длительность СД1, лет & $6,8(3,5-10,3)$ \\
\hline
\end{tabular}

Примечание. Данные представлены в виде медианы и интерквартильного размаха: Me [Q1; Q3].

с помощью критерия Манна-Уитни. В случае множественных сравнений использовалась поправка Бонферрони. Различие между качественными признаками оценивалось с помощью точного критерия Фишера. Значение р менее 0,05 считалось статистически значимым.

\section{РЕЗУЛЬТАТЬ}

Всего в исследование были включены 56 подростков, однако в процессе наблюдения 7 из них прервали свое участие в проекте по собственному желанию $(n=2)$ либо без объяснения причин $(n=5)$. Таким образом, во всех процедурах, предусмотренных протоколом, приняли участие 49 пациентов.
Клиническая характеристика пациентов, принявших участие в проекте, представлены в табл. 1.

Медиана длительности участия пациента в проекте составила 182 дня. За это время было проведено 266 дистанционных консультаций, что составило в среднем 5,4 консультации на одного участника, медиана продолжительности консультации составила 18 минут.

\section{Гликемический контроль}

Показатели $\mathrm{HbA}_{1 с}$ статистически значимо снизились уже к 12-й неделе исследования $(-0,3 \% ; \mathrm{p}=0,005)$, а к концу исследования изменение $\mathrm{HbA}_{1 с}$ относительно исходного уровня составило $-0,5 \%(p<0,001)$ (рис. 1$)$.

В отношении других показателей гликемического контроля также наблюдалась положительная динамика (табл. 2). Так, к концу исследования отмечалось статистически значимое увеличение процентов измерений глюкозы в целевом диапазоне 3,9-10,0 ммоль/л (+5,3 п.п.; $\mathrm{p}=0,016)$ и снижение вариабельности глюкозы крови, оцениваемое по показателю CV (-3,1 п.п.; $\mathrm{p}=0,015)$.

Значимое снижение среднего уровня $\mathrm{HbA}_{1 c}$ сопровождалось также увеличением числа и доли пациентов, достигнувших целевого уровня $\mathrm{HbA}_{1 c}<7,0 \%$, с 3 (6\%)

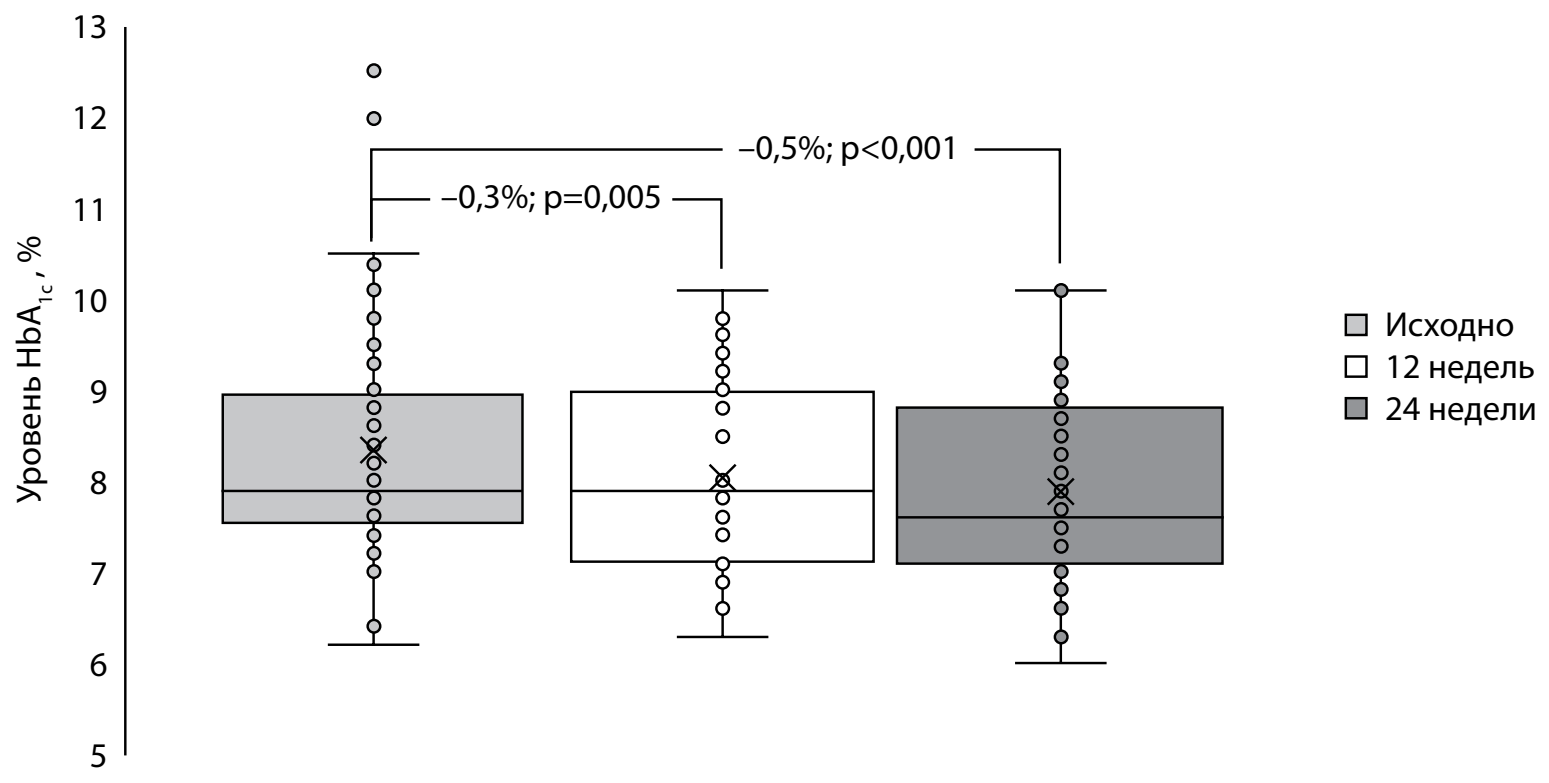

Рисунок 1.Динамикапоказателейгликированногогемоглобина.Данныепредставлены ввидемедианы (-), среднегозначения (х), интерквартильного размаха 25-75 ([]) и отдельных значений.

Таблица 2. Показатели гликемического контроля исходно, через 12 и 24 недели наблюдения

\begin{tabular}{|c|c|c|c|c|c|}
\hline Показатель & Исходно & 12 нед & 24 нед & P* & $\mathbf{P * *}$ \\
\hline Измерений в диапазоне 3,9-10 ммоль/л, \% & $49,7(38,3-62,5)$ & $56,3(44,8-62,3)$ & $57,2(44,6-69,6)$ & $\mathrm{H} / 3$ & 0,016 \\
\hline Измерений >10 ммоль/л, \% & $41,8(28,3-55,6)$ & $38,4(29,3-50,5)$ & $37,2(26,1-47,7)$ & $\mathrm{H} / 3$ & $\mathrm{H} / 3$ \\
\hline Измерений <3,9 ммоль/л, \% & $6(3,1-9,4)$ & $4,1(2-9,4)$ & $6(1,8-10)$ & $\mathrm{H} / 3$ & $H / 3$ \\
\hline Вариабельность гликемии, SD, ммоль/л & $3,9(3,3-4,8)$ & $3,6(2,9-4,7)$ & $3,7(2,9-4,7)$ & $\mathrm{H} / 3$ & $H / 3$ \\
\hline Вариабельность гликемии, CV, \% & $43,7(36,8-46,4)$ & $40,6(33,6-45,9)$ & $39,8(32,9-45,6)$ & $\mathrm{H} / 3$ & 0,015 \\
\hline
\end{tabular}

Примечание. Данные представлены в виде медианы и интерквартильного размаха: Мe [Q1; Q3].

* - уровень р при сравнении показателей исходно и через 12 нед, ** - уровень р при сравнении показателей исходно и через 24 нед. 


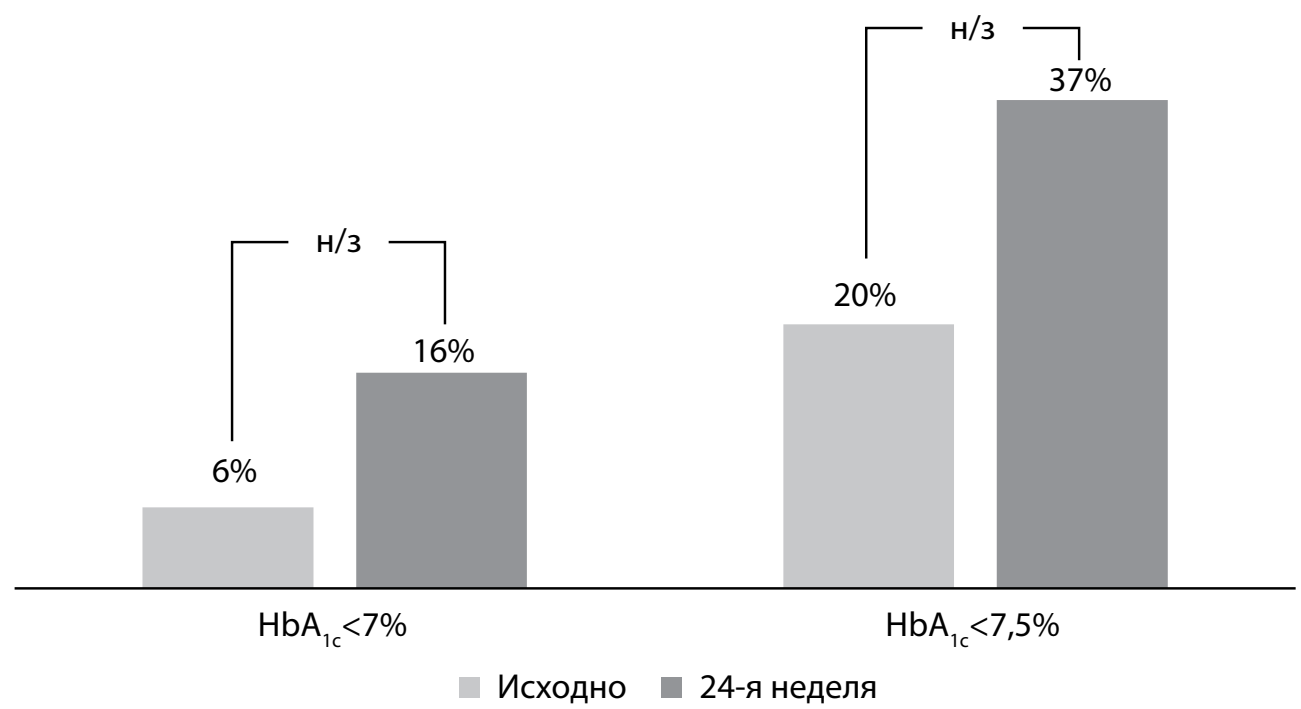

Рисунок 2. Доля пациентов, достигнувших показателя гликированного гемоглобина $<7,0$ и $<7,5 \%$, исходно и по окончании исследования (различия статистически не значимы).

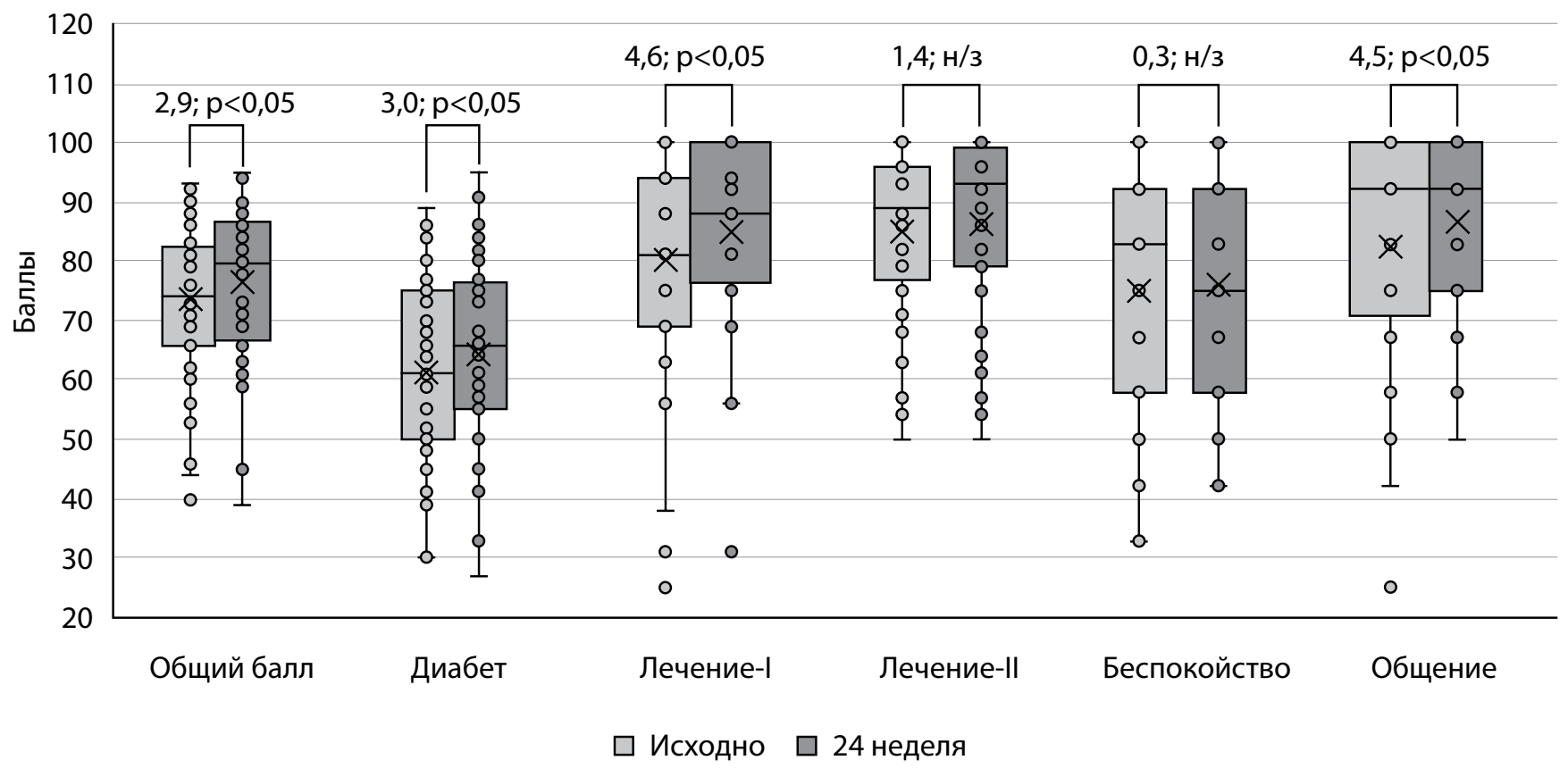

Рисунок 3. Показатели качества жизни PedsQL по окончании исследования по сравнению с исходным уровнем. Данные представлены в виде медианы (-), среднего значения (х), интерквартильного размаха 25-75 ([]) и отдельных значений.

до 8 (16\%) пациентов и <7,5\% с 10 (20\%) до 18 (37\%) пациентов, однако данные различия не были статистически значимыми (рис. 2).

\section{Качество жизни}

Результаты исследования качества жизни показали статистически значимое улучшение как совокупной оценки (общего балла) качества жизни пациентами (+2,9 балла; $p=0,008)$, так и отдельных составляющих ее показателей: отношение к диабету (шкала «Диабет» +3,0 балла; $\mathrm{p}=0,049$ ), отношение к лечению (шкала «Лечение-І» $+4,6$ балла; $p=0,010$ ) и общение с окружающими (шкала «Общение» +4,5 балла; $p=0,015$ ) (рис. 3).

\section{Результаты в зависимости от длительности} дистанционных консультаций

Принимая во внимание возможную зависимость эффекта дистанционного консультирования на гликемический контроль от длительности консультаций, а также влияние активного взаимодействия между врачом и пациентом на качество жизни, дополнительно была проведена оценка изменения показателей гликемического контроля и качества жизни у пациентов в зависимости от средней продолжительности дистанционной консультации: более и менее 20 минут (табл. 3).

Было установлено, что в группе пациентов со средней длительностью дистанционных консультаций >20 минут 
Таблица 3. Изменение показателей гликемического контроля и качества жизни в зависимости от средней продолжительности дистанционной консультации

\begin{tabular}{|c|c|c|c|}
\hline \multirow{3}{*}{ Показатель } & \multicolumn{2}{|c|}{ Изменение к 24-й неделе } & \multirow{3}{*}{$\mathbf{P}$} \\
\hline & \multicolumn{2}{|c|}{ средняя длительность консультации } & \\
\hline & $\begin{array}{c}>20 \text { мин } \\
(n=19)\end{array}$ & $\begin{array}{c}<20 \text { мин } \\
(n=30)\end{array}$ & \\
\hline \multicolumn{4}{|c|}{ Гликемический контроль } \\
\hline $\mathrm{HbA}_{1 c^{\prime}} \%$ & $-0,8(-1,9--0,2)$ & $-0,3(-0,5--0,1)$ & 0,049 \\
\hline Измерений в диапазоне 3,9-10 ммоль/л, \% & $7,6(-14,8-24,5)$ & $3,8(0,1-10,6)$ & $\mathrm{H} / 3$ \\
\hline Измерений > 10 ммоль/л, \% & $-8,5(-24,5-13,1)$ & $-1,7(-10,1-4,2)$ & $H / 3$ \\
\hline Измерений <3,9 ммоль/л, \% & $-0,1(-7,6-2,5)$ & $-1,5(-3,9-2,7)$ & $H / 3$ \\
\hline Вариабельность гликемии SD, ммоль/л & $-0,2(-0,6-0,5)$ & $-0,1(-0,6-0,1)$ & $H / 3$ \\
\hline Вариабельность гликемии CV, \% & $-3,2(-9,5--0,8)$ & $-2,2(-5,2-3,2)$ & $\mathrm{H} / 3$ \\
\hline \multicolumn{4}{|c|}{ Качество жизни } \\
\hline Общий балл & $4,4(0,9-8,1)$ & $4,5(-0,9-8)$ & $\mathrm{H} / 3$ \\
\hline Диабет & $6,8(-2,3-11,4)$ & $4,5(-4,5-13,6)$ & $\mathrm{H} / 3$ \\
\hline Лечение-І & $0(-6,3-6,3)$ & $6,3(0-12,5)$ & $H / 3$ \\
\hline Лечение-II & $0(0-7,1)$ & $0(-3,6-3,6)$ & $H / 3$ \\
\hline Беспокойство & $0(-8,3-8,3)$ & $0(-8,3-0)$ & $H / 3$ \\
\hline Общение & $0(-8,3-0)$ & $0(0-16,7)$ & $\mathrm{H} / 3$ \\
\hline
\end{tabular}

Таблица 4. Нежелательные явления. Данные представлены в виде: частота развития - количество и доля пациентов среди всех участников; случаи - число эпизодов среди всех участников и частота событий на одного пациента в год

\begin{tabular}{lcc|ccc}
\hline \multicolumn{1}{c}{ Показатель } & \multicolumn{2}{c|}{ Исходно } & \multicolumn{2}{c}{ Исследование } \\
\cline { 2 - 5 } & $\begin{array}{c}\text { частота } \\
\text { развития, } \mathbf{n}(\%)\end{array}$ & $\begin{array}{c}\text { случаи } \\
\text { (частота) }\end{array}$ & $\begin{array}{c}\text { частота } \\
\text { развития, } \mathbf{n}(\%)\end{array}$ & $\begin{array}{c}\text { случаи } \\
\text { (частота) }\end{array}$ \\
\hline Гипергликемии с кетозом & $3(6,1 \%)$ & $3(0,27)$ & $5(10,2 \%)$ & $5(0,21)$ & $\mathrm{H} / 3$ \\
Диабетический кетоацидоз & $1(2 \%)$ & $1(0,09)$ & $0(0 \%)$ & $0(0,00)$ & $\mathrm{H} / 3$ \\
Тяжелая гипогликемия & $2(4,1 \%)$ & $6(0,53)$ & $9(18,4 \%)$ & $16(0,67)$ & $\mathrm{H} / 3$ \\
\hline
\end{tabular}

отмечалось статистически значимо более выраженное снижение уровня $\mathrm{HbA}_{1 \mathrm{c}}$ к концу исследования, которое составило -1,0\% по сравнению с 0,2\% в группе пациентов с длительностью консультаций <20 минут. При этом между данными группами пациентов не наблюдалось статистически значимых различий в показателях качества жизни.

\section{Оценка удобства дистанционного консультирования}

и мобильного приложения ОT Reveal пациентами

и врачами

Результаты проведенного опроса подростков с СД1 и врачей представлены на рис. 4 и 5. Большинство врачей и пациентов положительно (не менее чем на 4 балла) оценили преимущества и удобство дистанционного консультирования с использованием мобильного приложения OT Reveal, его положительное влияние на уровень знаний по вопросам СД и показатели глюкозы, также подростки высоко оценили приватность и комфорт такого взаимодействия. Порядка $90 \%$ врачей и пациентов среди основных преимуществ дистанционного консультирования отметили возможность быстрой связи между врачом и пациентом.

\section{Острые осложнения СД1}

Частота нежелательных явлений (кетоз, диабетический кетоацидоз, тяжелая гипогликемия) значимо не изменилась в процессе исследования по сравнению с исходным уровнем (табл. 4).

\section{ОБСУЖДЕНИЕ}

Результаты исследования позволяют сделать вывод о достаточной эффективности дистанционного консультирования с использованием мобильного приложения OT Reveal в группе подростков с СД1 в отношении как показателей гликемического контроля, так и качества жизни.

В нашем исследовании использование мобильного приложения OT Reveal позволило достигнуть снижения $\mathrm{HbA}_{1 с}$ на 0,5\%, что является клинически значимым эффектом [8]. Принимая во внимание сложности, связанные с подростковым возрастом, которые приводят к ухудшению гликемического контроля, в том числе в результате ограниченного взаимодействия с лечащим врачом из-за дефицита времени, вопросов приватности и др., использование телемедицины демонстрирует 
По сравнению с бумажным дневником, данные самоконтроля из мобильного приложения являются более достоверными и полными

По сравнению с бумажным дневником/глюкометром, анализировать данные самоконтроля из мобильного приложения намного легче и быстрее

Анализ результатов пациента из мобильного приложения не требует много времени

Мобильное приложение позволило мне быть более эффективным при оценке гликемического контроля моих пациентов

Сложностей при дистанционном консультировании не возникало

Дистанционное консультирование в рамках проекта было удобным

Дистанционное консультирование способствует улучшению гликемического контроля

Дистанционное консультирование в обучении подростков навыкам управления диабетом эффективно

Участие в программе помогло подросткам занять более активную роль в управлении своим диабетом

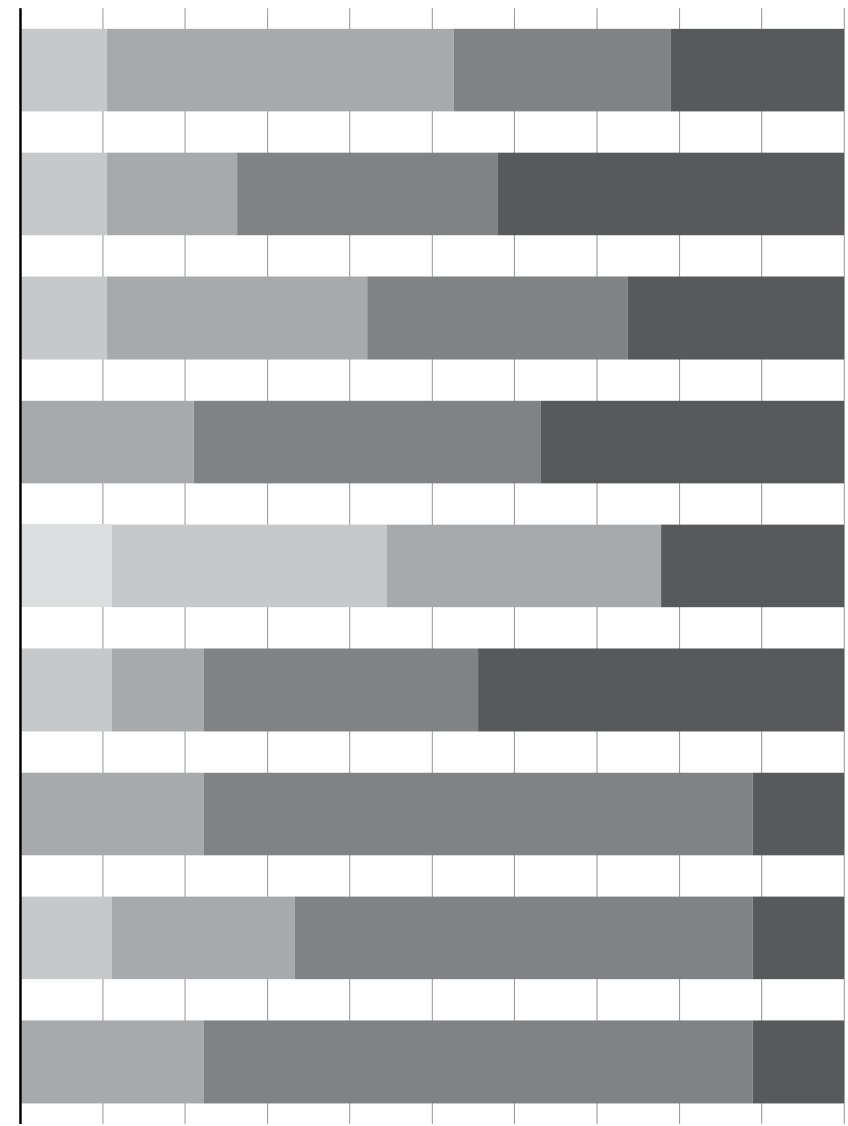

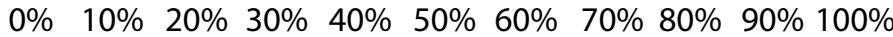

\section{1 балл 2 балла 3 балла 4 балла 5 баллов}

Возможность быстрой связи с подростком является важным преимуществом программы

Дистанционное консультирование - более комфортная форма обсуждения вопросов управления сахарным диабетом по сравнению с обычной очной консультацией

Хотел(-а) бы и дальше осуществлять дистанционное консультирование подростков

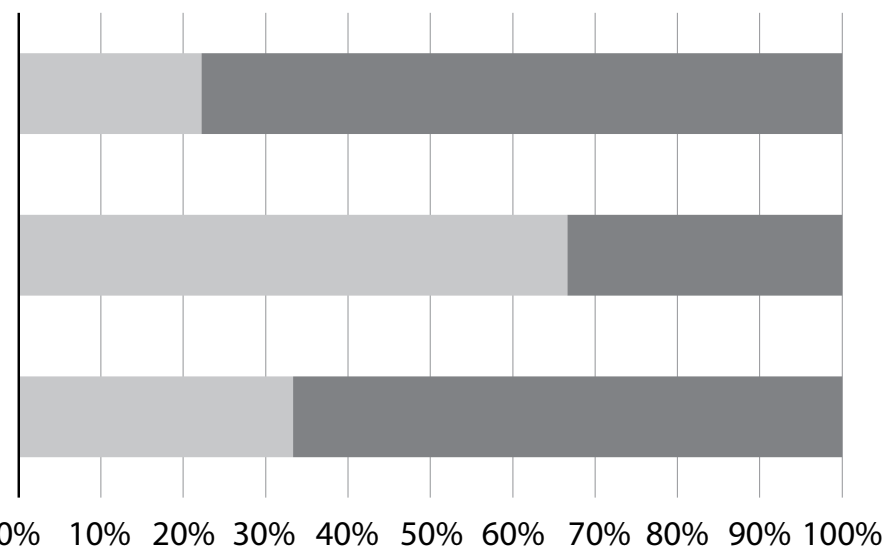

Нет Да

Сколько времени Вы затрачивали в среднем на анализ данных из мобильного приложения?

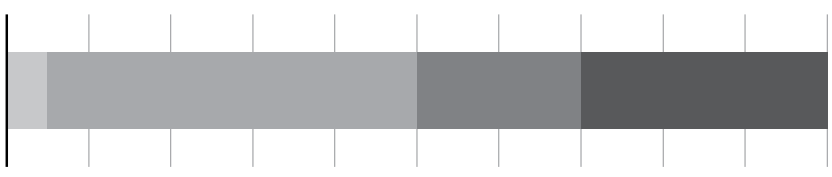

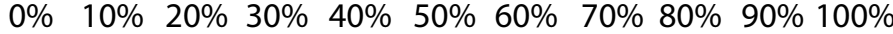

менее 5 мин от 5 до 10 мин более 10 мин Ш Друое время 
Посоветую друзьям с сахарным диабетом участвовать в подобной программе

Хотел/а бы и в дальнейшем иметь возможность дистанционной консультации с врачом

Считаю полезной возможность дистанционного консультирования с врачом без участия родителей

Сложностей при использовании мобильного приложения/платформы дистанционного консультирования не было

Дистанционные консультации с врачом были удобны

Благодаря программе знания по вопросам сахарного диабета значительно улучшены

Участие в программе помогло улучшить показатели глюкозы крови

$\begin{array}{llllllllllll}0 \% & 10 \% & 20 \% & 30 \% & 40 \% & 50 \% & 60 \% & 70 \% & 80 \% & 90 \% & 100 \%\end{array}$

\section{1 балл 2 балла 3 балла 4 балла 5 баллов}

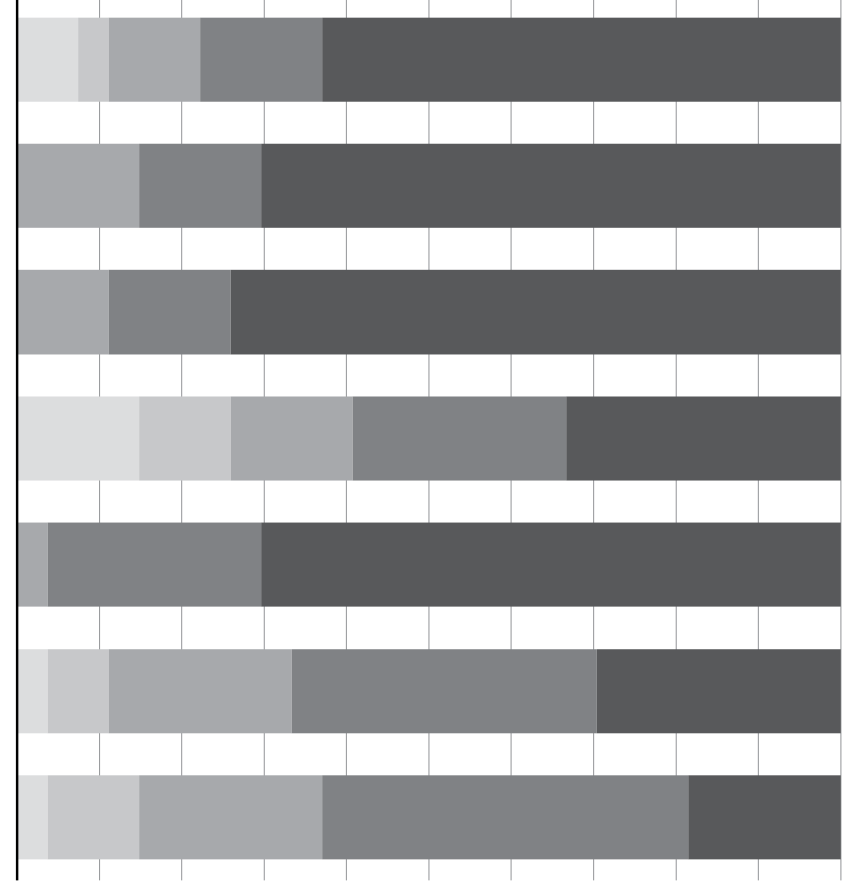

Возможность быстрой связи с врачом по телефонной/ интернет-связи является для меня очень важной

Дистанционное консультирование эндокринолога для меня более полезно для обсуждения вопросов управления сахарным диабетом по сравнению с обычной консультацией в поликлинике

Дистанционное консультирование эндокринолога для меня более комфортно для обсуждения вопросов управления сахарным диабетом по сравнению с обычными консультациями в поликлинике

Нет

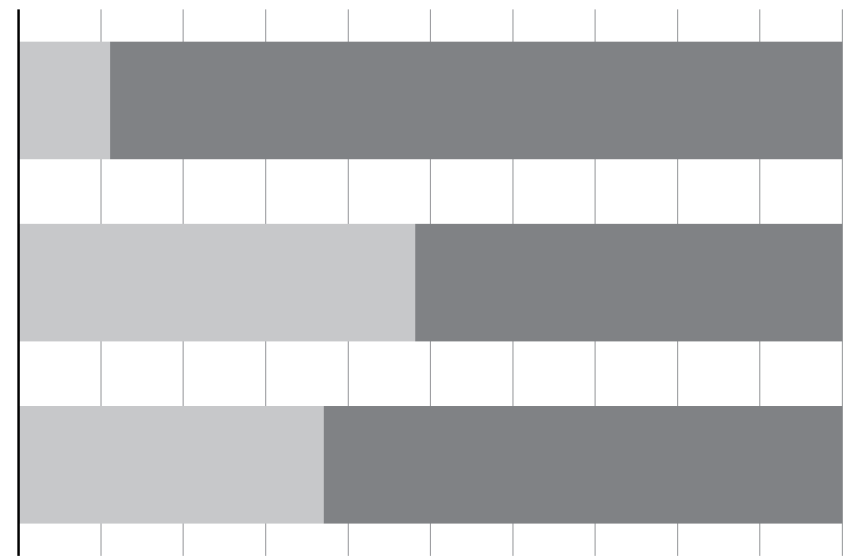

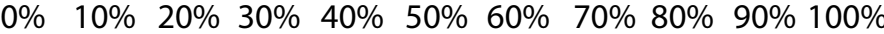

Ва Да

Рисунок 5. Оценка программы дистанционного консультирования и мобильного приложения (подростки).

возможности заполнить возникающие пробелы. Данные с носимых устройств для лечения СД1 (инсулиновые помпы, системы НМГ, глюкометры) могут быть считаны с использованием программного обеспечения производителя, что позволяет пациентам и родителям визуализировать данные, просматривать сводные статистические результаты и анализировать тенденции. Это также позволяет врачам просматривать такие данные дистанционно между визитами и делать более частые корректировки дозировки. Регулярная загрузка и просмотр данных о гликемии связаны со значительно более низким $\mathrm{HbA}_{1 с}$ (7,2\% против 8,1\%; $\left.\mathrm{p}=0,03\right)$ [9].

Телемедицина может помочь уменьшить различия при лечении диабета, особенно в отдаленных регионах, за счет улучшения доступа к медицинской помощи и снижения затрат на медицинскую помощь. Результаты систематического обзора с метаанализом указывают на то, что применение телемедицины у подростков может быть эффективным со средней разницей в $\mathrm{HbA}_{1 c^{\prime}}$ равной $-0,32 \%$, по сравнению с традиционной схемой наблюдения [10]. Интересно, что большую результативность продемонстрировали исследования с большей продолжительностью (не менее 6 мес) и те, в которых были пациенты с более высокими исходными значениями $\mathrm{HbA}_{1 c}(\geq 9 \%)$.

Результаты проведенного опроса свидетельствуют о высоком принятии дистанционного консультирования как среди врачей, так и среди подростков. Среди очевидных преимуществ дистанционного консультирования для врачей и пациентов можно выделить 
удобство и быстроту взаимодействия. Мобильное приложение OT Reveal позволяет вести пациенту электронный дневник и формировать структурированные отчеты, которые позволяют врачу наглядно оценить проводимое лечение и сформировать необходимые рекомендации. Очевидное удобство взаимодействия, доступность и приватность консультаций лечащего врача, а также положительное влияние на показатели глюкозы в совокупности способствуют, вероятно, повышению качества жизни подростков, что немаловажно в данном возрасте в связи со снижением данного показателя в результате воздействия как самого по себе сахарного диабета, так и других психосоциальных факторов [3]. Снижение качества жизни у пациентов с СД1 сопряжено с худшим гликемическим контролем, и наоборот, повышение уровня качества жизни может сопровождаться лучшей компенсацией диабета [3, 11]. Кроме того, использование сложных технологических и организационных решений при лечении СД1 у подростков может негативно отразиться на качестве жизни и, следовательно, на принятии таких подходов $[12,13]$, что придает большей значимости результатам этой работы.

Более активное взаимодействие между врачом и пациентом, как показано в нашей работе, может способствовать большей эффективности дистанционного консультирования без ущерба качеству жизни пациента. На данную взаимосвязь указывают и другие работы. Например, показано, что отправка лечащим врачом пациентам большего количества текстовых сообщений с рекомендациями сопровождается более выраженным снижением уровня $\mathrm{HbA}_{1 c}(1,0 \%$ против 0,5\%; $\mathrm{p}<0,05)$ [14]. Эти обстоятельства указывают на важность тесного контакта между врачом и пациентом и необходимость поиска и формирования оптимального алгоритма при дистанционном наблюдении.

Частота нежелательных явлений (тяжелая гипогликемия, ДКА, кетоз) была высокой как исходно, так и за время исследования, однако значимо не изменилась, что указывает на безопасность применяемого подхода при наблюдении подростков с СД1. В целом увеличение частоты острых осложнений СД1 не является ожидаемым явлением при использовании телемедицины [7, 10].

\section{ЗАКЛЮЧЕНИЕ}

Дистанционное консультирование с использованием мобильного приложения является эффективным в отношении гликемического контроля и качества жизни подходом у подростков с СД1, применение которого обеспечивает удобство и быстроту взаимодействия, а для подростков - еще и необходимый уровень комфорта и приватности.

Существенный процент отказа от участия в исследовании и высокий риск острых осложнений СД1 среди участников в очередной раз указывают на сложность ведения данной категории пациентов и важность оптимального контроля сахарного диабета, что также определяет необходимость формирования соответствующей среды оказания медицинской помощи и поддержки данной категории пациентов [3].

Клинически значимое улучшение показателей гликемии на фоне сохранения и повышения качества жизни при использовании технологий дистанционного консультирования свидетельствует о потенциальной перспективности широкого применения телемедицины у подростков с СД1.

\section{ДОПОЛНИТЕЛЬНАЯ ИНФОРМАЦИЯ}

Источник финансирования. Работа выполнена в рамках национальной благотворительной программы помощи детям с эндокринными заболеваниями «Альфа-Эндо» при финансовой поддержке фонда «КАФ». Компания ООО «ЛайфСкан Раша» предоставила для исследования тест-полоски и глюкометры.

Конфликт интересов. Авторы декларируют отсутствие явных и потенциальных конфликтов интересов, связанных с публикацией настоящей статьи.

Благодарность. Авторы выражают благодарность участникам и их семьям, исследователям, а также спонсору — фонду «КАФ» за финансовую поддержку в проведении этого исследования.

Информация о вкладе каждого автора. Лаптев Д.Н. - концепция и дизайн исследования, анализ полученных данных, написание текста; Еремина И.А. - сбор и обработка материала, редактирование текста; Карпушкина А.В. - концепция и дизайн исследования; Петряйкина Е.Е. - концепция исследования, сбор материала; Безлепкина О.Б. - концепция и дизайн исследования; Петеркова В.А. — концепция исследования, редактирование текста.

\section{СПИСОК ЛИТЕРАТУРЫ | REFERENCES}

1. Mayer-Davis EJ, Kahkoska AR, Jefferies C, et al. ISPAD Clinical Practice Consensus Guidelines 2018: Definition, epidemiology, and classification of diabetes in children and adolescents. Pediatr Diabetes. 2018;19:7-19. doi: https://doi.org/10.1111/pedi.12773

2. Дедов И.И., Шестакова М.В., Петеркова В.А. и др. Сахарный диабет у детей и подростков по данным Федерального регистра Российской Федерации: динамика основных эпидемиологических характеристик за 2013-2016 гг. // Сахарный диабет. - 2017. - Т. 20. — №6. C. 392-402. [Dedov II, Shestakova MV, Peterkova VA, et al. Diabetes mellitus in children and adolescents according to the Federal diabetes registry in the Russian Federation: dynamics of major epidemiological characteristics for 2013-2016. Diabetes mellitus. 2017;20(6):392-402. (In Russ.)]. doi: https://doi.org/10.14341/DM9460
3. Cameron FJ, Garvey K, Hood KK, et al. ISPAD Clinical Practice Consensus Guidelines 2018: Diabetes in adolescence. Pediatr Diabetes. 2018;19:250-261. doi: https://doi.org/10.1111/pedi.12702

4. White M, Sabin MA, Magnussen CG, et al. Long term risk of severe retinopathy in childhood-onset type 1 diabetes: a data linkage study. Med JAust. 2017;206(9):398-401. doi: https://doi.org/10.5694/mja16.00712

5. Trawley S, Browne JL, Hagger VL, et al. The Use of Mobile Applications Among Adolescents with Type 1 Diabetes: Results from Diabetes MILES Youth — Australia. Diabetes Technol Ther. 2016;18(12):813-819. doi: https://doi.org/10.1089/dia.2016.0233

6. Wu Y, Yao X, Vespasiani G, et al. Mobile App-Based Interventions to Support Diabetes Self-Management: A Systematic Review of Randomized Controlled Trials to Identify Functions Associated with Glycemic Efficacy. JMIR mHealth uHealth. 2017;5(3):e35. doi: https://doi.org/10.2196/mhealth.6522 
7. Лаптев Д.Н., Емельянов А.О., Самойлова Ю.Г., и др. Дистанционное наблюдение и лечение детей и подростков с сахарным диабетом 1 типа // Проблемы Эндокринологии. 2020. - T. 66. — №4. - C. 50-60. [Laptev DN, Emelyanov $A O$, Samoilova YG, et al. Remote monitoring and treatment of children and adolescents with type 1 diabetes. Problems of Endocrinology. 2020;66(4):50-60. (In Russ.)]. doi: https://doi.org/10.14341/probl12201

8. Little RR, Rohlfing $C L$. The long and winding road to optimal HbA1c measurement. Clin Chim Acta. 2013;418:63-71. doi: https://doi.org/10.1016/j.cca.2012.12.026

9. Wong JC, Neinstein AB, Spindler M, Adi S. A Minority of Patients with Type 1 Diabetes Routinely Downloads and Retrospectively Reviews Device Data. Diabetes Technol Ther. 2015;17(8):555-562. doi: https://doi.org/10.1089/dia.2014.0413

10. Lee SWH, Ooi L, Lai YK. Telemedicine for the Management of Glycemic Control and Clinical Outcomes of Type 1 Diabetes Mellitus: A Systematic Review and Meta-Analysis of Randomized Controlled Studies. Front Pharmacol. 2017:8:330. doi: https://doi.org/10.3389/fphar.2017.00330
11. Lee SWH, Ooi L, Lai YK. Children and Adolescents: Standards of Medical Care in Diabetes-2021. Diabetes Care. 2021:44(S1):180-199. doi: https://doi.org/10.2337/dc21-S013

12. Лаптев Д.Н., Переверзева С.В., Емельянов А.О., Петеркова В.А Мониторинг применения помповой инсулинотерапии у детей, подростков и молодых пациентов с сахарным диабетом 1 типа в Российской Федерации // Проблемы Эндокринологии. 2018. - T. 64. — №2. — C. 85-92. [Laptev DN, Pereverzeva SV, Emelyanov AO, Peterkova VA. Monitoring of insulin pump therapy in children, adolescents, and young adults with type 1 diabetes mellitus in the Russian Federation. Problems of Endocrinology. 2018;64(2):8592. (In Russ.)]. doi: https://doi.org/10.14341/probl8756

13. Lee SWH, Ooi L, Lai YK. Diabetes Technology: Standards of Medical Care in Diabetes-2021. Diabetes Care. 2021;44(S1):85-99. doi: https://doi.org/10.2337/dc21-S007

14. Grady M, Katz LB, Cameron H, et al. Diabetes App-Related Text Messages From Health Care Professionals in Conjunction With a New Wireless Glucose Meter With a Color Range Indicator Improves Glycemic Control in Patients With Type 1 and Type 2 Diabetes: Randomized Controlled Trial. JMIR Diabetes. 2017;2(2):e19. doi: https://doi.org/10.2196/diabetes.7454

\section{ИНФОРМАЦИЯ ОБ АВТОРАХ [AUTHORS INFO]}

*Лаптев Дмитрий Никитич, д.м.н. [Dmitry N. Laptev, MD, PhD]; адрес: Россия, 117036, Москва, ул. Дм. Ульянова, д. 11 [address: 11 Dm. Ulyanova street, 117036 Moscow, Russia]; ORCID: https://orcid.org/0000-0002-4316-8546;

elibrary SPIN: 2419-4019; e-mail: laptevdn@ya.ru

Еремина Ирина Александровна, к.м.н. [lrina A. Eremina, PhD]; ORCID: https://orcid.org/0000-0001-7021-1151; eLibrary SPIN: 9411-4710; e-mail: ieremina58@gmail.com

Карпушкина Анна Викторовна, д.м.н. [Anna V. Karpushkina, MD, PhD]; eLibrary SPIN: 2925-2033;

e-mail: akarpushkina@cafrussia.ru

Петряйкина Елена Ефимовна, д.м.н. [Elena E. Petryaykina, MD, PhD]; ORCID: https://orcid.org/0000-0002-8520-2378; eLibrary SPIN: 5997-7464; e-mail: lepet_morozko@mail.ru

Безлепкина Ольга Борисовна, д.м.н. [Olga B. Bezlepkina, MD, PhD]; ORCID: https://orcid.org/0000-0001-9621-5732; eLibrary SPIN: 3884-0945; e-mail: olgabezlepkina@mail.ru

Петеркова Валентина Александровна, д.м.н., профессор, академик PAH [Valentina A. Peterkova, MD, PhD, Professor]; ORCID: https://orcid.org/0000-0002-5507-4627; elibrary SPIN: 4009-2463; e-mail: peterkovava@hotmail.com

\section{ЦИТИРОВАТЬ:}

Лаптев Д.Н., Еремина И.А., Карпушкина А.В., Петряйкина Е.Е., Безлепкина О.Б., Петеркова В.А. Дистанционное наблюдение подростков с сахарным диабетом 1 типа с использованием мобильного приложения // Сахарный диабет. 2021. — T. 24. — №5. — C. 404-413. doi: https://doi.org/10.14341/DM12776

\section{TO CITE THIS ARTICLE:}

Laptev DN, Eremina NA, Karpushkona AV, Petryaykina EE, Bezlepkina OB, Peterkova VA. Remote monitoring of adolescents with type 1 diabetes mellitus using a mobile application. Diabetes Mellitus. 2021;24(5):404-413. doi: https://doi.org/10.14341/DM12776 